\title{
Stochastic Coupling and Thermodynamic Inequalities ${ }^{\star}$
}

\author{
Lawrence E. Thomas
}

Department of Mathematics, University of Virginia Charlottesville, VA 22903, USA

\begin{abstract}
Let $\mu_{1}$ and $\mu_{2}$ be thermodynamic Gibbs measures on $\mathbb{R}^{m}$ and $\mathbb{R}^{n}$, respectively. Diffusions are constructed having $\mu_{1}$, and $\mu_{2}$ as invariant measures. These diffusions are then coupled; inequalities between expectations of certain random variables on the two spaces result.
\end{abstract}

\section{Introduction}

Let $\left(X, \mu_{1}\right)$ and $\left(Y, \mu_{2}\right)$ be two probability measure spaces and let $\phi_{1}, \ldots, \phi_{k}$ and $\psi_{1}, \ldots, \psi_{k}$ be families of random variables on $X$ and $Y$ respectively. Then we say $\mu_{1}$ is stochastically less than $\mu_{2}$ with respect to $\phi_{1}, \ldots, \phi_{k} ; \psi_{1}, \ldots, \psi_{k}$, and write $\mu_{1} \stackrel{\S}{\leqq} \mu_{2}$, if for all $\left(\lambda_{1}, \ldots, \lambda_{k}\right) \in \mathbb{R}^{k}, \mu_{1}\left\{\phi_{1} \geqq \lambda_{1}, \ldots, \phi_{k} \geqq \lambda_{k}\right\} \leqq \mu_{2}\left\{\psi_{1} \geqq \lambda_{1}, \ldots\right.$, $\left.\psi_{k} \geqq \lambda_{k}\right\}$. Note that if $\mu_{1} \stackrel{\S}{\leqq} \mu_{2}$ and $f$ is an increasing function on $\mathbb{R}, \int f\left(\phi_{j}\right) d \mu_{1} \leqq$ $\int f\left(\psi_{j}\right) d \mu_{2}$, from integration by parts; stochastic inequality of measures allows one to compare expectations of certain random variables on $X$ and $Y$. The purpose of this article is to describe conditions under which this stochastic comparison can be made, in the setting where $\mu_{1}$ and $\mu_{2}$ are thermodynamic Gibbs measures on $\mathbb{R}^{m}$ and $\mathbb{R}^{n}$ and $\phi_{1}, \ldots, \phi_{k}$ and $\psi_{1}, \ldots, \psi_{k}$ are linear functions on $\mathbb{R}^{m}$ and $\mathbb{R}^{n}$, respectively.

The basic ideas of the paper are illustrated in the simple example of $d \mu_{1}(x)=$ $\exp \left(-H_{1}(x)\right) d x, d \mu_{2}(y)=\exp \left(-H_{2}(y)\right) d y$ both probability measures on $\mathbb{R}$, $\phi(x)=x, \psi(y)=y$, and $H_{1}$ and $H_{2}$ smooth. Let $K=\frac{1}{2}\left(\nabla_{x}+\nabla_{y}\right)^{2}-\frac{1}{2}\left(\nabla_{x} H_{1}\right) \nabla_{x}-$ $\frac{1}{2}\left(\nabla_{y} H_{2}\right) \nabla_{y}$ be a differential operator acting on continuous functions on $\mathbb{R}^{2}$. (From an operator standpoint, the "coupling" is the cross term $\nabla_{x} \nabla_{y}$ in $K$.) Under suitable conditions, $K$ is the generator of a semigroup $\exp (t k)$ representable by $\exp (t K) f\left(x_{0}, y_{0}\right)=E f\left(x\left(t, x_{0}\right), y\left(t, y_{0}\right)\right)$ with $E$ expectation with respect to Brownian motion and $\left(x\left(t, x_{0}\right), y\left(t, y_{0}\right)\right)$ the solution to a coupled set of stochastic differential equations. Suppose $\nabla_{x} H_{1}(x) \geqq \nabla_{x} H_{2}(x)$. Then these equations yield a stochastic differential inequality which implies that $x\left(t, x_{0}\right) \leqq y\left(t, y_{0}\right)$ if the initial values for $x\left(t, x_{0}\right), y\left(t, y_{0}\right)$ satisfy $x_{0} \leqq y_{0}$. Let $\hat{\mu}$ be a probability measure on $\mathbb{R}^{2}$ supported in the region $x \leqq y$ and let $g_{1}(x, y)=f(x), g_{2}(x, y)=f(y)$ with $f$ a continuous

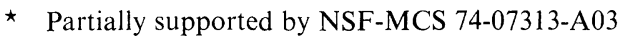


increasing function on $\mathbb{R}$. Then for $t>0$

$$
\int d \hat{\mu} \exp t K g_{1} \leqq \int d \hat{\mu} \exp t K g_{2} \text {. }
$$

On the other hand, restricted to functions of $x$ only, $\exp K$ "collapses" to the "marginal" semigroup $\exp \frac{t}{2}\left(\Delta_{x}-\left(\nabla_{x} H_{1}\right) \nabla_{x}\right)$ which, under suitable hypotheses, is ergodic with invariant measure $\mu_{1}$. Similar remarks hold for $\exp t K$ restricted to functions of $y$. Taking the limit $t \rightarrow \infty$ of ineq. (1.1), we obtain

$$
\int f(x) d \mu_{1}(x) \leqq \int f(y) d \mu_{2}(y)
$$

which implies $\mu_{1} \leqq \mu_{2}$. (Note that the condition $\nabla_{x} H_{1}(x) \geqq \nabla_{x} H_{2}(x)$ means the second force $F_{2} \equiv-\nabla_{x} H_{2}$ exceeds the first force $F_{1} \equiv-\nabla_{x} H_{1}$; one might expect the second measure to have its mass shifted to the right, relative to the first measure. Indeed, this is the case.)

The main theorem concerning stochastic comparison of measures is given in Sect. 2. Not too surprisingly, the conditions on the measures are closely related to those of the FKG correlation inequalities. In the last section a version of these inequalities is derived as a corollary of the theorem. The last section also contains a comparison of a measure on $\mathbb{R}^{m}$ with a one-dimensional Gaussian measure and a remark on extending the theorem to manifolds.

It is appropriate to mention that there is substantial literature on the study of statistical mechanical states via stochastic models having these states as their invariant measures, e.g. Holley and Stroock's work on the stochastic Ising model, [1], and Holley [2]. See also the fine survey article by Liggett [3]. Recently, Faris [4] has studied diffusions on manifolds having the Gibbs state for the classical Heisenberg model as invariant measure. Finally, we point out that the strategy used here to prove the theorem of Sect. 2 is in part an adaptation to the continuous case of a coupling argument employed by Holley in [2] to prove a discrete version of the FKG inequalities.

I am particularly indebted to Loren Pitt for many useful discussions.

\section{Stochastic Comparison of Thermodynamic Measures}

In the following, $x$ will denote a point in $\mathbb{R}^{m}, y$ a point in $\mathbb{R}^{n}$. Let $d \mu_{1}(x)=e^{-H_{1}(x)} d x$, $d \mu_{2}(x)=e^{-H_{2}(y)} d y$ be probability measures on $\mathbb{R}^{m}$ and $\mathbb{R}^{n}$ respectively. The main result is the following.

Theorem. Assume $H_{1}, H_{2}$ are $C^{\infty}$ functions tending to $\infty$ for their respective arguments tending to $\infty$, and satisfying $\nabla H_{i} \cdot \nabla H_{i}-2 \Delta H_{i} \geqq c, i=1,2$ for some constant $c$. Let $\phi_{1}(x), \phi_{2}(x), \ldots, \phi_{k}(x)$ be $k$ real linear functions on $\mathbb{R}^{m}, \psi_{1}(x)$, $\psi_{2}(x), \ldots, \psi_{k}(x), k$ real linear functions on $\mathbb{R}^{n}$, and assume there is a unitary transformation $C$ from span $\left\{\nabla \psi_{1}, \ldots, \nabla \psi_{k}\right\} \subset \mathbb{R}^{n}$ to span $\left\{\nabla \phi_{1}, \ldots, \nabla \phi_{k}\right\} \subset \mathbb{R}^{m}$ such that $C \nabla \psi_{i}=\nabla \phi_{i} i=1,2, \ldots, k$. Suppose further that on each hyperplane $S_{i}$ in $\mathbb{R}^{m} \times \mathbb{R}^{n}$ defined by

$$
\begin{aligned}
& S_{i}=\left\{(x, y) \in \mathbb{R}^{m} \times \mathbb{R}^{n} \mid \phi_{i}(x)=\psi_{i}(y)\right\}, \\
& \nabla \phi_{i} \cdot \nabla H_{1}(x) \geqq \nabla \psi_{i} \nabla H_{2}(y)
\end{aligned}
$$


holds where $S_{i}$ intersects $\mathbf{U} \equiv\left\{(x, y) \in \mathbb{R}^{m} \times \mathbb{R}^{n} \mid \phi_{j}(x) \leqq \phi_{j}(y), j=1, \ldots, k\right\}$. Finally, suppose $\mathbf{U}$ has non-empty interior. Then $\mu_{1} \stackrel{s}{\leqq} \mu_{2}$ with respect to $\phi_{1}, \ldots, \phi_{k} ; \psi_{1}, \ldots, \psi_{k}$ in the sense that

$$
\mu_{1}\left\{\phi_{1} \geqq \lambda_{1}, \ldots, \phi_{k} \geqq \lambda_{k}\right\} \leqq \mu_{2}\left\{\psi_{1} \geqq \lambda_{1}, \ldots, \psi_{k} \geqq \lambda_{k}\right\}
$$

for all $\left(\lambda_{1}, \ldots, \lambda_{k}\right) \in \mathbb{R}^{k}$. If $f$ is a bounded function on $\mathbb{R}^{k}$, increasing in each of its variables, then

$$
\int f\left(\phi_{1}, \ldots, \phi_{k}\right) d \mu_{1} \leqq \int f\left(\psi_{1}, \ldots, \psi_{k}\right) d \mu_{2} .
$$

The smoothness condition of the $H_{i}$ 's can be relaxed somewhat. In particular, if the $H_{i}$ 's are limits of smooth $H^{\prime}$ 's satisfying the hypotheses, the conclusion of the theorem will still hold. Note that $\left\{\nabla \phi_{1}, \ldots, \nabla \phi_{k}\right\}$ may be linearly dependent and $k$ may, in fact, exceed $m, n$.

Proof. The proof first involves construction of a coupled process, and then completion of the proof.

Step 1. The coupling. Extend the matrix $C$ to all of $\mathbb{R}^{n}$ (regarded as a vector space) by setting it equal to zero on the orthogonal complement of span $\left\{\nabla \psi_{i}\right\}$ continuing to call the extension $C$. Let $A$ be the (positive) $(m+n) \times(m+n)$ matrix.

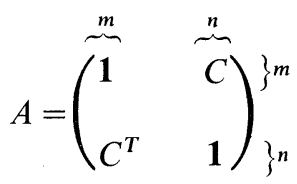

and let

$$
\Delta_{s}=\sum_{i, j}^{m+n} A_{i j} \partial_{i} \partial_{j}
$$

where $\partial_{i}=\partial / \partial x_{i} i \leqq m, \partial_{i}=\partial / \partial y_{i-m} i>m$. A priori $\Delta_{s}$ is a quadratic in tangent and normal vectors to the 1 -codimensional surface $S_{i}$ in $\mathbb{R}^{m} \times \mathbb{R}^{n}$ defined in the statement of the theorem but since $\sum_{l}^{m+n} A_{j l} \partial_{l}$ annihilates $\phi_{i}-\psi_{i} i=1,2, \ldots, k, \Delta_{s}$ in fact involves tangent vectors only and acts invariantly in $S_{i}$ (and translates of $\left.S_{i}\right)$. Let $S_{0}=\left\{(x, y) \in \mathbb{R}^{m} \times \mathbb{R}^{n} \mid \nabla \phi_{i} \cdot x=\nabla \psi_{i} \cdot y, i=1,2, \ldots, k\right\}$.

Let $\left\{\exp \frac{1}{2} t \Delta_{s}\right\}$ be the semigroup acting in $C\left(\mathbb{R}^{m+n}\right)$ (continuous functions with bounded sup-norm) generated by $\Delta_{s}$. Let $W(t)=\left(w_{1}(t), w_{2}(t)\right) \in S_{0} \subset \mathbb{R}^{m} \times \mathbb{R}^{n}$ be the associated (degenerate) Brownian motion originating at the origin. Now $\exp \frac{1}{2} t \Delta_{s}$, restricted to functions depending only on $x$ (resp. $y$ ), reduces to exp $\frac{1}{2} t \Delta_{1}$ (resp. $\left.\exp \frac{1}{2} t \Delta_{2}\right)$ with $\Delta_{1}, \Delta_{2}$ the Laplacians on $\mathbb{R}^{m}$, (resp. $\left.\mathbb{R}^{n}\right)$, as follows from the definition of $\Delta_{s}$. Moreover the components of $W(t)=\left(w_{1}(t), w_{2}(t)\right)$ are just normal $m$ and $n$ dimensional Brownian motions respectively, which, however, are coupled in the sense that $W(t)$ stays in $S_{0}$.

Let $X\left(t, x_{0}, y_{0}\right)=(x(t), y(t))$ be the solution to the coupled set of stochastic 
differential equations

$$
d X(t)=\left(\begin{array}{l}
d x(t) \\
d y(t)
\end{array}\right)=-\frac{1}{2}\left(\begin{array}{l}
\nabla H_{1}(x(t)) \\
\nabla H_{2}(y(t))
\end{array}\right) d t+\left(\begin{array}{l}
d w_{1}(t) \\
d w_{2}(t)
\end{array}\right)
$$

with $X(t)$ originating at $\left(x_{0}, y_{0}\right)$. (The existence, continuity and uniqueness of $X(t)$ is outlined in the appendix, lemma (A.1).) Since $W$ lies in $S_{0}, d W=\left(d w_{1}, d w_{2}\right)$ is perpendicular to the constant vector $\left(\nabla \phi_{i},-\nabla \psi_{i}\right)$ for each $i$, thus

$$
\begin{aligned}
& d\left(\phi_{i}(x(t))-\psi_{i}(y(t))\right) \\
& \quad=d\left(\left(\nabla \phi_{i^{\prime}}-\nabla \psi_{i}\right) \cdot X(t)\right)=-\frac{1}{2}\left(\nabla \phi_{i} \cdot \nabla H_{1}(x(t))-\nabla \psi_{i} \cdot \nabla H_{2}(y(t))\right) d t,
\end{aligned}
$$

which is negative by Eq. (2.1) on the surface $S_{i}$ in the region where $S_{i}$ intersects U. It follows that if $X(t)$ starts at a point $\left(x_{0}, y_{0}\right) \in \mathbf{U} \equiv\left\{(x, y) \mid \phi_{j}(x) \leqq \psi_{j}(y) j=\right.$ $1,2, \ldots, k\}$, then $\phi_{j}(x(t)) \leqq \psi_{j}(y(t))$ for all $t \geqq 0$ so that $X\left(t, x_{0}, y_{0}\right) \in \mathbf{U}$.

Step 2. The Coupled Semigroup and Completion of the Proof. Let $K^{t}$ be the operator acting in $C\left(\mathbb{R}^{(m+n)}\right)$ defined by

$$
K^{t} f\left(x_{0}, y_{0}\right)=E f\left(X\left(t, x_{0}, y_{0}\right)\right)
$$

with $E$ expectation with respect to $W$. Let $f$ be a bounded continuous increasing function on $\mathbb{R}^{k}$ in the sense $f\left(z_{1}, \ldots, z_{k}\right) \leqq f\left(z_{1}^{\prime}, \ldots, z_{k}^{\prime}\right)$ if $z_{1} \leqq z_{1}^{\prime}, \ldots, z_{k} \leqq z_{k}^{\prime}$, and set $f \circ \phi(x)=f\left(\phi_{1}(x), \ldots, \phi_{k}(x)\right), f \circ \psi(y)=f\left(\psi_{1}(y), \ldots, \psi_{k}(y)\right)$ (regarding them as functions on $\left.\mathbb{R}^{m+n}\right)$. Finally, let $h(x, y)$ be a bounded positive continuous function on $\mathbb{R}^{m+n}$ satisfying $\int h(x, y) d \mu_{1}(x) d \mu_{2}(y)=1$ and which is supported in the region $\mathbf{U}$ defined in the theorem. (This is possible since $\mathbf{U}$ has non-empty interior).

Now if $X(t)$ starts in $\mathbf{U}$, it stays in $\mathbf{U}$ by the last paragraph of Step 1, so that by eqn. $(2-8)$ and the increasing property of $f$,

$$
\int h(x, y) K^{t} f \circ \phi(x) d \mu_{1}(x) d \mu_{2}(y) \leqq \int h(x, y) K^{t} f \circ \psi(y) d \mu_{1}(x) d \mu_{2}(y) .
$$

On the other hand, $K^{t}$ restricted to functions of $x$ (resp. $y$ ) only reduces to $P_{1}^{t}=\exp \frac{t}{2}\left(\Delta_{1}-\nabla H_{1} \cdot \nabla\right)\left(\right.$ resp. $\left.P_{2}^{t}=\exp \cdot \frac{t}{2}\left(\Delta_{2}-\nabla H_{2} \cdot \nabla\right)\right)$, by Eq. (2.6) and propositions (A.2), (A.3) of the Appendix. Inequality (2.9) becomes

$$
\int\left(\int h(x, y) d \mu_{2}(y)\right) P_{1}^{t} f \circ \phi(x) d \mu_{1}(x) \leqq \int\left(\int h(x, y) d \mu_{1}(x)\right) P_{2}^{t} f \circ \psi(y) d \mu_{2}(y) .
$$

Now both $P_{1}^{t}, P_{2}^{t}$ are self-adjoint contraction semigroups in the Hilbert spaces $L^{2}\left(\mathbb{R}^{m}, d \mu_{1}\right), L^{2}\left(\mathbb{R}^{n}, d \mu_{2}\right)$ respectively and both have unique ground states given by the constant function (see Proposition (A.3)). Taking the limit $t \rightarrow \infty$, we obtain, by Proposition (A.4),

$$
\int f \circ \phi(x) d \mu_{1}(x) \leqq \int f \circ \psi(y) d \mu_{2}(y) .
$$

To obtain ineq. (2.3) for arbitrary $f$, we simply apply (2.11) to a sequence of increasing continuous functions $\left\{f_{n}\right\}$ converging (weakly) to $f$. Ineq. (2.2) follows by choosing $f$ to be the indicator function for $z_{1} \geqq \lambda_{1}, \ldots, z_{k} \geqq \lambda_{k}$. This concludes the proof of the theorem. 


\section{Application and Remarks}

\section{Example 1. Comparison with Gaussians}

Let $B$ be a real positive definite $m \times m$ matrix, $\hat{\phi}$ a real eigenvector of $B$ with eigenvalue $\varepsilon$, and normalized in the sense $\sum_{l}^{m} \hat{\phi}(l)^{2}=1$. Let $d \mu_{1}(x)=e^{-H_{1}(x)} d x$ be a probability measure on $\mathbb{R}^{m}$ with $H_{1}(x)=\frac{1}{2} \sum_{l, l^{\prime}}^{m} B_{l, l^{\prime}} x_{l} x_{l^{\prime}}+V(x)$ and $V$ such that $\sum_{l}^{m} \hat{\phi}(l) \nabla_{l} V(x) \geqq 0$. Let $\phi(x)=\sum_{l}^{m} \hat{\phi}(l) x_{l}$. Let $d \mu_{2}(y)=\sqrt{\frac{\varepsilon}{2 \pi}} \exp -\varepsilon / 2 y^{2} d y$ be a Gaussian measure on $\mathbb{R}$ so that $H_{2}(y)=\frac{\varepsilon}{2} y^{2}+$ const and set $\psi(y)=y$. Then $\nabla \phi \cdot \nabla H_{1}(x) \geqq \varepsilon \phi(x)=\nabla \psi \cdot \nabla H_{2}(y)$ for $\phi(x)=\psi(y),|\nabla \phi|=|\nabla \psi|$, so that by the theorem $\mu_{1} \leqq \mu_{2}$ with respect to $\phi, \psi$. If $f$ is increasing, $\int f(\phi(x)) d \mu_{1}(x) \leqq$ $\int f(y) d \mu_{2}(y)$.

\section{Remark 2. FKG Inequalities}

There is a connection between the main theorem and FKG inequalities [cf. 5]. The following is a version of these inequalities.

Corollary 3.1. Suppose in addition to the smoothness and boundedness assumption of the theorem, $H(x)$ on $\mathbb{R}^{m}$ satisfies

$$
\frac{\partial H}{\partial x_{i}}\left(x_{1}, \ldots, x_{i-1}, x_{i}, x_{i+1}, \ldots, x_{m}\right) \geqq \frac{\partial H}{\partial x_{i}}\left(x_{1}^{\prime}, \ldots, x_{i-1}^{\prime}, x_{i}, x_{i+1}^{\prime}, \ldots, x_{m}^{\prime}\right)
$$

for $x_{1} \leqq x_{1}^{\prime}, \ldots, x_{i-1} \leqq x_{i-1}^{\prime}, x_{i+1} \leqq x_{i+1}^{\prime}, \ldots, x_{m} \leqq x_{m}^{\prime}$, for each $i, 1 \leqq i \leqq m$. Then if $f$ and $g$ are increasing on $\mathbb{R}^{m}$,

$$
\langle f g\rangle-\langle f\rangle\langle g\rangle \geqq 0
$$

with $\langle\cdot\rangle=\int(\cdot) e^{-H} d x \equiv \int(\cdot) d \mu$.

Proof. Assume first that $f$ is smooth and bounded. For $\varepsilon \geqq 0$, let $H_{\varepsilon}(x)=H(x)-$ $\varepsilon f(x)+c(\varepsilon)$ with $c(\varepsilon)$ adjusted so that $d \mu_{\varepsilon}(x)=\exp \left(-H_{\varepsilon}(x)\right) d x$ is a probability measure. Let $\phi_{i}(x)=\psi_{i}(x)=x_{i} i=1,2, \ldots, m$. Then inequality (3.1) implies $\nabla \phi_{i} \cdot \nabla H(x) \geqq \nabla \psi_{i} \cdot \nabla H_{\varepsilon}(y)$ on each surface $\phi_{i}(x)=\psi_{i}(y)$ in $\mathbb{R}^{2 m}$ for $\phi_{j}(x) \leqq \psi_{j}(y)$, $j \neq i$, so that by the theorem $\mu \leqq \mu_{\varepsilon}$ and if $g$ is increasing then

$$
\int g d \mu_{\varepsilon} \geqq \int g d \mu .
$$

Dividing this inequality by $\varepsilon$ and taking the limit $\varepsilon \rightarrow 0$, we obtain (3.2) for smooth $f$. For arbitrary $f$ we simply apply (3.2) to a sequence $\left\{f_{n}\right\}$ of smooth increasing functions converging (weakly) to $f$. 


\section{Remark 3. Measures on Manifolds}

The strategy of proof of the theorem perhaps can be extended to obtain comparison of measures on manifolds. A heuristic result in this direction is:

Proposition (3.2). Let $d \mu_{1}=\exp \left(-H_{1}\right) d \Omega^{v}, d \mu_{2}=\exp \left(-H_{2}\right) d \Omega^{v^{\prime}}$ be probability measures on $v$ and $v^{\prime}$ dimensional sphere $S^{v}, S^{v^{\prime}}$ respectively, $v, v^{\prime} \geqq 2$, with $H_{1}\left(\Omega^{v}\right)$, $H_{2}\left(\Omega^{v^{\prime}}\right)$ smooth. Let $\theta, \theta^{\prime}$ be the colatitudes on the respective spheres. Then $\mu_{1} \leqq \mu_{2}$ in the sense $\mu_{1}(\theta \geqq \lambda) \leqq \mu_{2}\left(\theta^{\prime} \geqq \lambda\right)$ for $0 \leqq \lambda \leqq \pi$ if

$$
\frac{\partial}{\partial \theta}\left(H_{1}-\left(v-v^{\prime}\right) \ln \sin \theta\right) \geqq \frac{\partial}{\partial \theta^{\prime}} H_{2}
$$

for all $\Omega^{\nu}, \Omega^{\nu^{\prime}}$ such that $\theta=\theta^{\prime}$.

Outline of Proof. In order to set up suitable diffusions, it is convenient to first map the spheres to cylinders by $\Omega^{v}=\left(\theta, \Omega^{v-1}\right) \rightarrow\left(z, \Omega^{v-1}\right), \Omega^{v^{\prime}}=\left(\theta^{\prime} \Omega^{v^{\prime}-1}\right) \rightarrow$ $\left(z^{\prime}, \Omega^{\nu^{\prime}-1}\right)$ with $z=-\cot \theta, z^{\prime}=-\cot \theta^{\prime}$. The respective measures become $d \hat{\mu}_{1}\left(z, \Omega^{\nu-1}\right)=\exp -\left(H_{1}+(v+1) \ln \left(1+z^{2}\right)^{1 / 2}\right) d z d \Omega^{v-1}$, and a similar expression for $d \mu_{2}$. (The exp $-(v+1) \ln \left(1+z^{2}\right)^{1 / 2}$ factor is a Jacobian.) Suitable diffusion processes on the cylinders can then be defined, corresponding to semigroup generators $G_{1}, G_{2}$ with

$$
G_{1}=\frac{1}{2}\left(\frac{\partial^{2}}{\partial z^{2}}+\Delta_{s^{v-1}}\right)-\frac{1}{2}\left(\frac{\partial H_{1}}{\partial z}+\frac{(v+1) z}{\left(1+z^{2}\right) 1 / 2}\right) \frac{\partial}{\partial z}-\frac{1}{2} \nabla_{s^{v-1}} H_{1} \cdot \nabla_{s^{v-1}},
$$

and a similar expression for $G_{2}\left(\Delta_{s^{\nu-1}}\right.$ is the Laplacian on $\left.S^{\nu-1}\right)$. The diffusion processes are solutions to stochastic differential equations involving Brownian motion on the cylinders; they are coupled by identifying the axial components of Brownian motion on the two cylinders. The remaining argument is analogous to that used in proving the theorem.

\section{Appendix}

We review briefly some technical aspects of the semigroup generators encountered in the text. Most of the results here are well known and are a straight forward adaptation of [6]. Let $d \mu(x)=\exp (-H(x)) d x$ be a probability measure on $\mathbb{R}^{m}$ and let $w$ be normal $m$-dimensional Brownian motion originating at the origin. Let $x(t)$ be the solution, if it exists, to

$$
x(t)=x_{0}-\frac{1}{2} \int_{0}^{t} \nabla H(x(s)) d s+w(t) .
$$

If we wish to make the initial value for $x(t)$ explicit, we write $x\left(t, x_{0}\right)$.

Lemma (A.1). Suppose that $H(x)$ is $C^{\infty}, \nabla H \cdot \nabla H-\Delta H$ is bounded below on $\mathbb{R}^{m}$, and that $H(x) \rightarrow \infty, x \rightarrow \infty$. Then $x(t)$ exists, is continuous, and is unique for almost every $w(\cdot)$.

Proof. The local existence, continuity, and uniqueness of $w(t)$ follows from a contraction mapping argument [6], since $\nabla H$ satisfies local Lipshitz condition. 
By Ito's lemma [7], the differential of $H(x(t))$ is

$d H(x(t))=\frac{1}{2}(-\nabla H \cdot \nabla H(x(t))+\Delta H(x(t))) d t+\nabla H(x(t)) \cdot d w \leqq c d t+\nabla H(x(t)) d w$

for some constant $c$. Taking the (Brownian motion) expectation of this inequality, we obtain

$$
\frac{d}{d t} E(H(x(t))) \leqq c
$$

implying $E(H(x(t)))$ is finite, hence $x(t)$ exists a.s.

Let $C\left(\mathbb{R}^{m}\right)$ be the Banach space of continuous functions on $\mathbb{R}^{m}$ in the sup norm, and define $P^{t} f\left(x_{0}\right)=E f\left(x\left(t, x_{0}\right)\right)$ with $x\left(t, x_{0}\right)$ the solution to eqn. (A.1).

Proposition A.2. Let $H$ satisfy the hypotheses of lemma (A.1). Then the family $\left\{P^{t}\right\}$ is a contraction semigroup on $C\left(\mathbb{R}^{m}\right)$. The generator $G$ of $P^{t}$ restricted to functions in $C_{0}^{\infty}\left(\mathbb{R}^{m}\right)$, is given by

$$
G f(x)=\frac{1}{2}(\Delta-\nabla H \cdot \nabla) f(x) .
$$

Proof. Continuity of $P^{t} f(x)$ in $x$ follows from the fact that $x\left(t, x_{1}\right) \rightarrow x\left(t, x_{0}\right)$ for $x_{1} \rightarrow x_{0}$ a.s. and a dominated convergence argument. Moreover $P^{t}$ is clearly contractive so that $P^{t}: C\left(\mathbb{R}^{m}\right) \rightarrow C\left(\mathbb{R}^{m}\right)$. The semigroup property of $\left\{P^{t}\right\}$ follows from the Markov property for $x(t)$.

Let $f \in C_{0}^{\infty}\left(\mathbb{R}^{m}\right)$. Then by Ito's lemma [7],

$$
\begin{aligned}
\frac{1}{t} & \left(P^{t} f\left(x_{0}\right)-f\left(x_{0}\right)\right)=\frac{1}{t} E \int_{0}^{t} d f(x(s)) \\
= & \frac{1}{2 t} E\left(\int_{0}^{t}\left(-\nabla f \cdot\left(\nabla H(x(s)) d s+d_{w}\right)+\nabla f(x(s)) d s\right)\right) \\
= & \frac{1}{2 t} E\left(\int_{0}^{t}(-\nabla f \cdot \nabla H(x(s))+\Delta f(x(s))) d s\right)
\end{aligned}
$$

which converges to $G f\left(x_{0}\right)$ for $t \rightarrow 0$ (by dominated convergence).

We conclude with some Hilbert space theory for $G$, defined by equation (A.2). Let $\mathscr{H}=L^{2}\left(\mathbb{R}^{m}, d \mu\right)$ with inner product $\langle,\rangle_{\mathscr{H}}$.

Proposition (A.3). Assume that $H$ satisfies the hypothesis of lemma $(A . I)$ and, in addition, assume that $(\nabla H)^{2}-2 \Delta H$ is bounded below. Then $G$, regarded as a symmetric operator on $\mathscr{H}$ is essentially self-adjoint on $C_{0}^{\infty}\left(\mathbb{R}^{m}\right)$. The spectrum of $G$ is contained in $(-\infty, 0]$ The operator $G$ has a unique ground state given by the constant function $f_{0}(x) \equiv 1$ with corresponding eigenvalue 0 . The semigroup generated by (the closure of ) $G$ agrees with $P^{t}$ on $C\left(\mathbb{R}^{m}\right)$.

Proof. Let $U: \mathscr{H} \rightarrow L^{2}\left(\mathbb{R}^{m}, d x\right)$ be the unitary map given by $U f(x)=$ $\exp \left(-\frac{1}{2} H(x)\right) f(x)$. Then $G^{\prime}=U G U^{-1}=\frac{1}{2}\left(\Delta-\frac{1}{8}(\nabla H)^{2}+\frac{1}{4} \Delta H\right)$, and, for example, by Kato's inequality [8] $G^{\prime}$ is essentially self adjoint on $C_{0}^{\infty}\left(\mathbb{R}^{m}\right)$. Hence $G$ must be essentially self adjoint.

Now $G$ has Dirichlet form $Q(f, g)=\langle\nabla f, \nabla g\rangle_{\mathscr{H}}$ implying that the spectrum of $G$ is negative. Any eigenfunction $f$ of $G$ is smooth by elliptic regularity so that $Q(f, f)<0$ unless $f$ is constant, which implies uniqueness of the ground state. 
To prove the final assertion is tedious. One begins by first defining a sequence of Hamiltonians $\left\{H_{n}\right\}$ converging to $H$, with each $H_{n}$ smooth and constant outside some ball of radius $n$. For each $n$ we define a process $x_{n}(t)$ (by Eq. (A.1) with $H$ replaced by $H_{n}$ ) and corresponding semigroup $P_{n}^{t}$. The generator $G_{n}$ of $P_{n}^{t}$ is clearly dissipative; Kato's inequality can be applied to $G_{n}$ to show, by the Hille-Yosida theorem, that $P_{n}^{t}$ considered as acting in $C_{0}\left(\mathbb{R}^{m}\right)$ is uniquely determined by the action of $G_{n}$ on $C^{\infty}\left(\mathbb{R}^{m}\right)$. Moreover $G_{n}$ is symmetric in $\mathscr{H}_{n}=L^{2}\left(\mathbb{R}^{m}, \exp \left(-H_{n}\right) d x\right)$ and, again by Kato's inequality, essentially self-adjoint on $C_{0}^{\infty}\left(\mathbb{R}^{m}\right)$. It follows that $\left\{P_{n}^{t}\right\}$ and the $L^{2}$-semigroup $\left\{\exp t \bar{G}_{n}\right\}$ can be identified on $C_{0}^{\infty}\left(\mathbb{R}^{m}\right)$. Since $P_{n}^{t} f(x)$ converges to $P^{t} f(x)$ (because $x^{n}(t) \rightarrow x(t)$ a.s.) and the $L^{2}$-semigroup $\exp t \bar{G}_{n}$ converge to $\exp t \bar{G}$ it follows that $P^{t}$ and $\exp t \bar{G}$ can be identified on $C_{0}^{\infty}\left(\mathbb{R}^{m}\right)$. Finally, for $f$ arbitrary in $C\left(\mathbb{R}^{m}\right)$, let $f_{n} \in C_{0}^{\infty}\left(\mathbb{R}^{m}\right)$ with $\left\|f_{n}\right\|_{C\left(\mathbb{R}^{m}\right)} \leqq\|f\|$ converge weakly to $f$. Then

$$
P^{t} f(x)=\lim _{n \rightarrow \infty} P^{t} f_{n}(x)=\lim _{n \rightarrow \infty} \exp t \bar{G} f_{n}(x)=\exp t \bar{G} f(x) .
$$

Proposition A.4. Let $H$ satisfy the hypothesis of proposition (A.3). Let $g \in \mathscr{H}$, $f \in C\left(\mathbb{R}^{m}\right)$. Then

$$
\lim _{t \rightarrow \infty}\left\langle g P^{t} f\right\rangle_{\mathscr{H}}=\langle g, 1\rangle_{\mathscr{H}}\langle 1, f\rangle_{\mathscr{H}}
$$

Proof. By the previous proposition $P^{t}$ can be identified with the contractive $L^{2}$-semigroup having unique ground state $f_{0} \equiv 1$. The result follows from application of the spectral theorem.

\section{References}

1. Holley, R. A., Stroock, D. W. : $\mathrm{L}_{2}$ theory for the stochastic Ising model. Z. Wahrscheinlichkeitstheorie verw. Gebiete 35, 87-101 (1976)

2. Holley, R. A. : Recent results on the stochastic Ising model. Rocky Mount. J. Math. 4, 479-496 (1974)

3. Liggett, T. M. : The stochastic evolution of infinite systems of interacting particles. In: École d'été de probabilités de Saint-Flour VI-1976, (ed. J. Hoffmann-Jorgensen), Lecture Notes in Mathematics 598. Berlin, Heidelberg, New York: Springer 1977

4. Faris, W. G. : The stochastic Heisenberg model. J. Functional Anal. 32, 343-352 (1979). See also The Rayleigh-Schrödinger expansion of the Gibbs state of a classical Heisenberg ferromagnet, preprint, Department of Mathematics, University of Arizona

5. Cartier, P. : Inégalités de corrélation en méchanique statistique in Séminaire Bourbaki, Vol. 1972/73. In: Lecture Notes in Mathematics 383, pp. 242-264. Berlin, Heidelberg, New York: Springer 1974

6. Nelson, E. : Dynamical theories of Brownian motion, particularly Chap. 8. Princeton, New Jersey: Princeton University Press 1967

7. McKean, H. P. Jr. : Stochastic integrals. New York: Academic Press 1969

8. Kato, T. : Schrödinger operators with singular potentials. Israel J. Math. 13, 135-148 (1972). See also Reed, M. Simon, B. : Methods of modern mathematical physics II, p. 184. New York: Academic Press 1975

Communicated by E. Lieb

Received February 27, 1980 